III Simpósio Paranaense de Modelagem.

Simulaçãoe Controle de Processos

ISSN : 1984-7521
Arigo:

22

Páginas: 160-167

\title{
INFLUÊNCIA DA RADIAÇÃO NA CONVECÇÃO NATURAL EM CAVIDADES TRAPEZOIDAIS CONTENDO DEFLETORES
}

\author{
Mariana Marques da Cunha ${ }^{1 *}$, Éliton Fontana ${ }^{1}$ \\ 1 - Universidade Federal do Paraná -UFPR - PR, mariana.cunha@ufpr.br
}

\begin{abstract}
Resumo - Interferência da radiação na convecção natural é estudada em cavidades trapezoidais, contendo dois obstáculos. A geometria da cavidade é composta de duas paredes isotérmicas, a da direita, que é mantida resfriada e a da esquerda, que é mantida aquecida, possui ainda uma superfície isolada no fundo e um topo inclinado. $O$ método de volumes finitos para a modelagem. Foram analisados três diferentes casos, primeiramente sem a presença da radiação, explorando somente a convecção natural; com presença da radiação somente na fase gasosa por último, considerando da radiação tanto no fluido quanto através dos sólidos que compõe os defletores. Foram analisadas situações para diferentes valores do número de Rayleigh $(R a)\left(10^{4} \leq R a \leq 10^{6}\right)$ e do número de Boltzmann $(B o)(1 \leq B o \leq 10)$, mostrando que a radiação altera significativamente os campos de velocidade $e$ temperatura no interior da cavidade. E que para baixos valores de Bo foi observada a estratificação no perfil de temperatura dos obstáculos.
\end{abstract}

Palavras-chave: Radiação, convecção natural, cavidades trapezoidais, defletores, modelagem matemática.

\section{Introdução}

A convecção é uma forma de transporte de calor, juntamente com a condução e a radiação, ocorre com o aparecimento de um gradiente de temperatura em um meio fluido fazendo com que ocorra o movimento deste. Essa forma de transferência de energia ocorre por meio de dois mecanismos, o microscópico, aleatório, em que ocorre a difusão das moléculas e o movimento macroscópico, do fluido em si. A união destes dois movimentos, havendo um gradiente de temperatura, permite a troca térmica. Dependendo do tipo de escoamento do fluido, a convecção pode ser dividida forçada ou natural. Quando o escoamento tem origem externa, por bombeamento ou ventilação, denomina-se convecção forçada. A convecção natural, também chamada de convecção livre, objeto de estudo neste artigo, ocorre quando o escoamento do fluido é gerado pela força de empuxo decorrida da diferença de densidades no fluido [1]. A diferença de densidade surge com variações na temperatura em seu interior. Pode-se exemplificar isso por meio de um fluido em contato com uma parede horizontal isotérmica, sendo essa mantida em uma temperatura superior ao do fluido, devido a ação da gravidade, o líquido aquecido, por possuir menor densidade, ascende, enquanto o fluido frio desce, gerando correntes de convecção e transportando energia em forma de calor [2].

A brisa marítima, a ebulição da água, o voo de um balão à ar quente, o cozimento do arroz, ar condicionados e aquecedores, a formação de nuvens, a movimentação de placas tectônicas, turbinas a vapor, trocadores de calor e a circulação sanguínea de animais endotérmicos são exemplos práticos da ocorrência da convecção. Devido à sua grande abrangência, essa forma de transmissão de calor é tema de muitos trabalhos e artigos científicos. No presente trabalho é investigada a convecção natural em cavidades trapezoidais, sendo que esta geometria tem importantes aplicações na área das engenharias, por abranger situações do cotidiano, como por exemplo determinados cômodos. Além disso, diversas vezes incorporada à cavidade existem objetos defletores, representando obstáculos encontrados na prática, como estudado por Silva et al [3], Moukalled et al [4] e também Fontana et al [5].

Dependendo da natureza dos defletores e das condições do sistema, a influência da troca térmica por radiação pode ser significativa, porém, a grande maioria dos trabalhos encontrados na literatura negligencia sua influência para reduzir o gasto computacional. A radiação, diferentemente da condução e da convecção não necessita de um meio material para transferir calor, ao contrário, no vácuo essa forma de transporte de calor é ainda mais eficiente [1]. Nela o 
calor é emitido por corpos que estejam em uma temperatura superior ao zero absoluto, através de ondas eletromagnéticas. Raios solares, lareiras e fogueiras, fornos e estufas são alguns exemplos de aplicação da radiação no cotidiano.

Tendo como base as duas formas de transporte de energia mencionadas anteriormente, o presente artigo propõe uma análise da influência da radiação na convecção natural em cavidades trapezoidais com dois refletores em seu interior. O tema da combinação das duas formas de transferência térmica já foi discutido por trabalhos na área, como Reynolds et al [6], que analisou combinação dessas duas formas de transporte de energia em cavidades trapezoidais, porém, sem a presença de defletores, e suas análises foram voltadas especificamente para a energia solar. Para uma melhor análise de como a radiação atua em uma cavidade trapezoidal contendo defletores, foi utilizada uma geometria semelhante à proposta por Silva et al [3] utilizando-se malhas e condições de contornos similares, adicionando no modelamento, a radiação, neste caso, ambos os defletores se encontram no chão, enquanto que no estudo de Fontana et al [5] foi estudado a posição alternada dos defletores, um no teto e o outro no chão.

Foram analisados três casos distintos. Primeiramente, foi considerando somente convecção. No segundo caso foi considerando convecção e a radiação somente para a fase gasosa, assumindo sólidos opacos. Por último, foram consideradas a convecção e a radiação tanto para a fase gasosa, quanto para os obstáculos, tratados como sólidos semitransparentes.

\section{Modelagem Matemática}

A geometria proposta é uma cavidade composta de duas paredes verticais limitadas por uma superfície inferior plana e uma superior inclinada, sendo a direita mantida resfriada, enquanto a esquerda é aquecida. As fronteiras inferior e a superior foram consideradas isoladas. No interior da cavidade, foram posicionados dois defletores de modo a parcialmente obstruir o fluxo. A posição e dimensão dos defletores seguiu o proposto por Adriano et al [2], conforme mostrado na Fig. 1. A altura da parede direita $(\mathrm{H})$ é quatro vezes menor que o comprimento da cavidade (L), o ângulo do teto $(\theta)$ foi considerado como $15^{\circ}$. Quanto aos defletores, com espessuras (W) equivalente a $1 / 20$ do comprimento, o primeiro e o segundo defletores foram posicionados, a uma distância de $1 / 3$ e $2 / 3$ do comprimento, respectivamente. Suas alturas foram fixadas como $2 \mathrm{H}^{*} / 3$, em que $\mathrm{H}^{*}$ é altura da cavidade na posição em que o defletor se encontra. A temperatura da parede, esquerda, indicada em vermelho na Fig. 1 , é $T_{H}$ ao mesmo tempo que a parede direita, indicada em azul, possui um temperatura fria $T_{C}$.

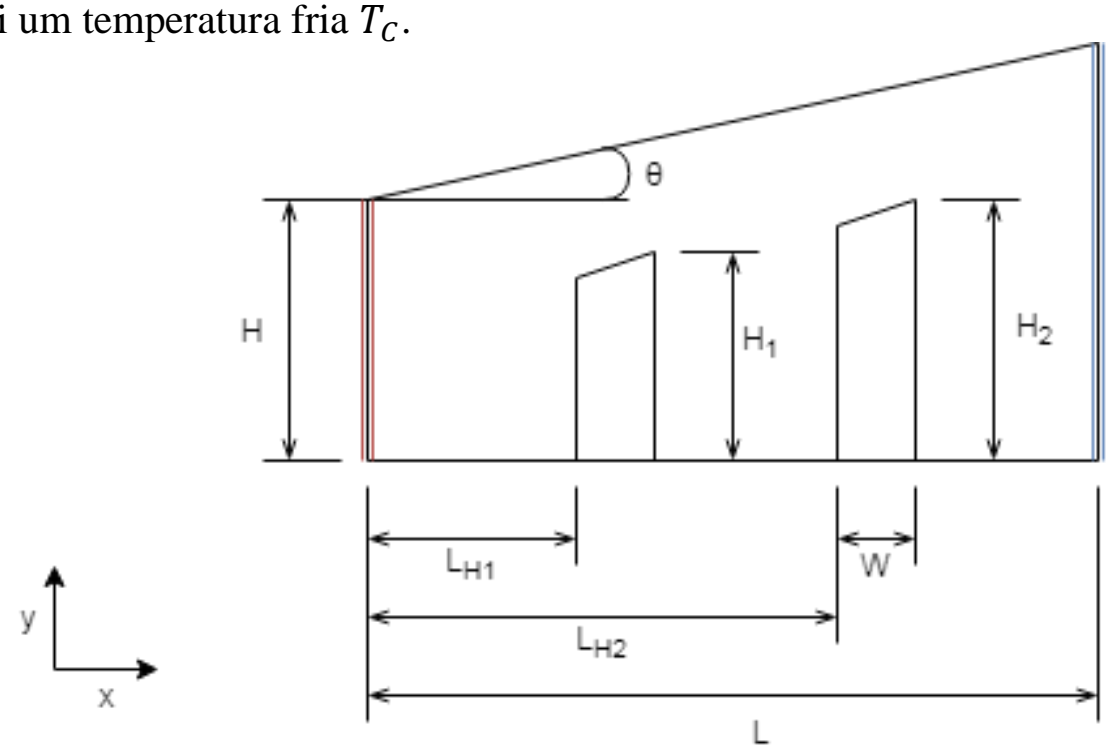

Figura 1 - Geometria proposta 
Excetuando-se pela densidade na direção y, onde atua a gravidade, as demais propriedades do fluido foram consideradas constantes, sendo este considerado o ar utilizando a aproximação de Boussinesq. Considerando um regime estacionário e laminar, as equações de conservação de massa, momento e energia na fase fluida são listadas a seguir:

$$
\begin{aligned}
& \nabla \cdot \vec{v}=0 \\
& \frac{1}{P r}(\vec{v} \cdot \nabla \vec{v})=-\nabla p+\nabla^{2} \vec{v}+R a \theta \vec{\jmath} \\
& \vec{v} \nabla \theta=\nabla^{2} \theta
\end{aligned}
$$

onde $\vec{v}=(u, v)$, sendo que $u$ e $v$ representam, respectivamente, as componentes (adimensionais) da velocidade na direção $x$ e $y, \rho$ representa a densidade do fluido, $p$ a pressão adimensional, $\beta$ a expansividade térmica, $\theta=\left(T-T_{C}\right) /\left(T_{H}-T_{C}\right)$ a temperatura adimensional, $\alpha$ a difusividade térmica e $\vec{\jmath}$ o vetor unitário na direção y. Os números adimensionais de Pradtl e Rayleigh são definidos como:

$$
\operatorname{Pr}=\frac{v}{\alpha} \quad R a=\frac{g \beta\left(T_{H}-T_{C}\right) H^{3}}{v \alpha}
$$

onde $v=\mu / \rho$ é a viscosidade cinemática, sendo $\mu$ a viscosidade dinâmica e $\rho$ a densidade do fluido, $\alpha=k / \rho c_{p}$ é a difusividade térmica, sendo $k$ e $c_{p}$ a condutividade e a capacidade calorífica da fase fluida, respectivamente. Neste trabalho, foi considerado que $\operatorname{Pr}=0.7$ (correspondendo ao ar ambiente) e diferentes valores de $R a$ foram avaliados.

Para os casos onde a radiação na fase sólida não é considerada, a conservação de energia na fase sólida resulta em:

$$
\nabla^{2} \theta=0
$$

As condições de contorno de temperatura prescrita nas superfícies esquerda (quente) e direita (fria) resultam em:

$$
\begin{aligned}
& \theta(x=0, y)=1 \quad \theta(x=L, y)=0 \\
& \begin{aligned}
u(x=0, y)= & v(x=0, y)=u(x=L, y)=v(x=L, y)=u(x, y=0) \\
& =v(x, y=0)=u(x, y=H+L * \operatorname{tg}(\theta)) \\
& =v(x, y=H+L * \operatorname{tg}(\theta))=0
\end{aligned}
\end{aligned}
$$

As superfícies isoladas termicamente resultam em:

$$
\left.\left.\frac{\partial \theta}{\partial y}\right)_{y=0}=\frac{\partial \theta}{\partial y}\right)_{y=H+L * \operatorname{tg}(\theta)}=0
$$

A continuidade do fluxo de calor na interface sólido/fluido pode ser expressa como:

$$
\hat{n} \cdot \vec{\nabla} \theta=(n \cdot \vec{\nabla} \theta) k_{r}
$$


onde $k_{r}$ é a razão entre a condutividade térmica da fase sólida e da fase fluida. No presente trabalho, considerou-se que $k_{r}=2$.

Para avaliar a influência da radiação, considerou-se dois modelos distintos, um para modelar a transferência através da fase gasosa, considerada como um meio transparente e outro para a transferência através do meio sólido, considerado como semitransparente. Para a fase gás, utilizou-se o modelo de transferência discreta. A ideia principal deste modelo é considerar que a radiação emitida pelas superfícies em um determinado intervalo de ângulo de superfície pode ser aproximada como um único raio. Este raio então viaja pelo domínio, sendo absorvido ou refletido pelas demais superfícies internas. Diferentes modelos foram avaliados para a fase gás e este apresentou melhor resultado.

Já para a fase sólida, foi utilizado o modelo de Monte-Carlo, sendo este um método estatístico que assume que a intensidade da radiação é proporcional ao fluxo angular diferencial de fótons, de modo que a intensidade média da radiação depende da distância viajada por um fóton e das propriedades do meio. Neste trabalho, o material sólido foi considerado como sendo um vidro a base de sílica, com índice de refração de 1.47012 , coeficiente de absorção de $0.001 \mathrm{~m}^{-1} \mathrm{e}$ coeficiente de espalhamento nulo. O gasto computacional associado ao modelo de Monte-Carlo é bastante elevado, sendo que para obter a precisão desejada foi necessário avaliar a um número de histórias de $5 \times 10^{5}$. Quando este modelo é utilizado, o tempo de processamento aumenta por um fator de mais de 150 vezes. As equações associadas aos modelos de transferências discreta e Monte-Carlo não serão apresentadas aqui, porém pode ser encontras em [7].

Para mensurar a importância da radiação em comparação com a convecção, foram avaliados casos para diferentes valores do número de Boltzmann, definido como:

$$
B o=\frac{\rho c_{p} u_{0}}{\sigma T_{m}{ }^{3}}
$$

onde $\sigma=5.67 \times 10^{-8} \mathrm{~W} / \mathrm{m}^{2} K^{4}$ é a constante de Stephan-Boltzmann, $T_{m}=\left(T_{H}+T_{C}\right) / 2$, indicando a temperatura média e $u_{0}=\sqrt{2 g \beta H T_{m}}$ é uma escala de velocidade associada com a convecção natural. Neste trabalho, foram considerados valores de $B o$ entre 1 e 10.

As equações foram resolvidas utilizando o pacote ANSYS CFX versão 16.0, software para simulações na área da engenharia, para a análise de fluidos, em que as equações são resolvidas simultaneamente, sendo considerado como critério de convergência quando a soma dos valores absolutos normalizados para cada célula da malha no domínio da solução atinge um valor inferior a $10^{-6}$.

\section{Resultados e Discussão}

Como comentado anteriormente, foram analisados três casos: sem radiação (Caso 1); considerando convecção e a radiação somente para a fase gasosa, utilizando o modelo de transferência discreta (Caso 2) e considerando convecção, condução e radiação tanto para a fase gasosa, quanto para os obstáculos, utilizando o modelo de Monte Carlo para a fase sólida (Caso $3)$.

Na Fig. 2 são apresentados os perfis de temperatura e as linhas de fluxo para o Caso 1, considerando diferentes valores de $R a$. Nesta e nas demais figuras, a distribuição de temperatura é apresentada em termos da temperatura adimensional $\theta$ e da velocidade normalizada $v^{*}=$ $v / v_{\max }$, onde $v$ é a componente vertical do vetor velocidade e $v_{\max }$ é o valor máximo de $v$ para o caso avaliado.

Considerando-se o primeiro caso, onde foi considerada somente a atuação da convecção, vide Fig. 2, averiguou-se que com o aumento do $R a$, também é amplificada a intensidade da 
convecção. Na Fig. 2-a, com $R a=10^{4}$, é possível notar a formação de isotermas predominantemente verticais, demonstrando a prevalência da condução como meio de transporte de calor, ao passo que para valores superiores de $R a$ tais linhas de temperatura tendem a posicionar-se principalmente na horizontal, como nas Fig. 2-b e Fig. 2-c, indicando desta maneira o predomínio da convecção ao mesmo tempo em que a transferência de energia através da condução é reduzida. Verifica-se uma dependência da convecção ao analisar-se o perfil de velocidade, observando-se as linhas de fluxo, para pequenos valores de $R a$, é possível identificar o surgimento de três regiões aproximadamente estagnadas (Fig. 2-a), à medida que, para valores mais elevados (Fig. 2-b e Fig. 2-c), constata-se uma maior circulação nas regiões intermediárias aos obstáculos.

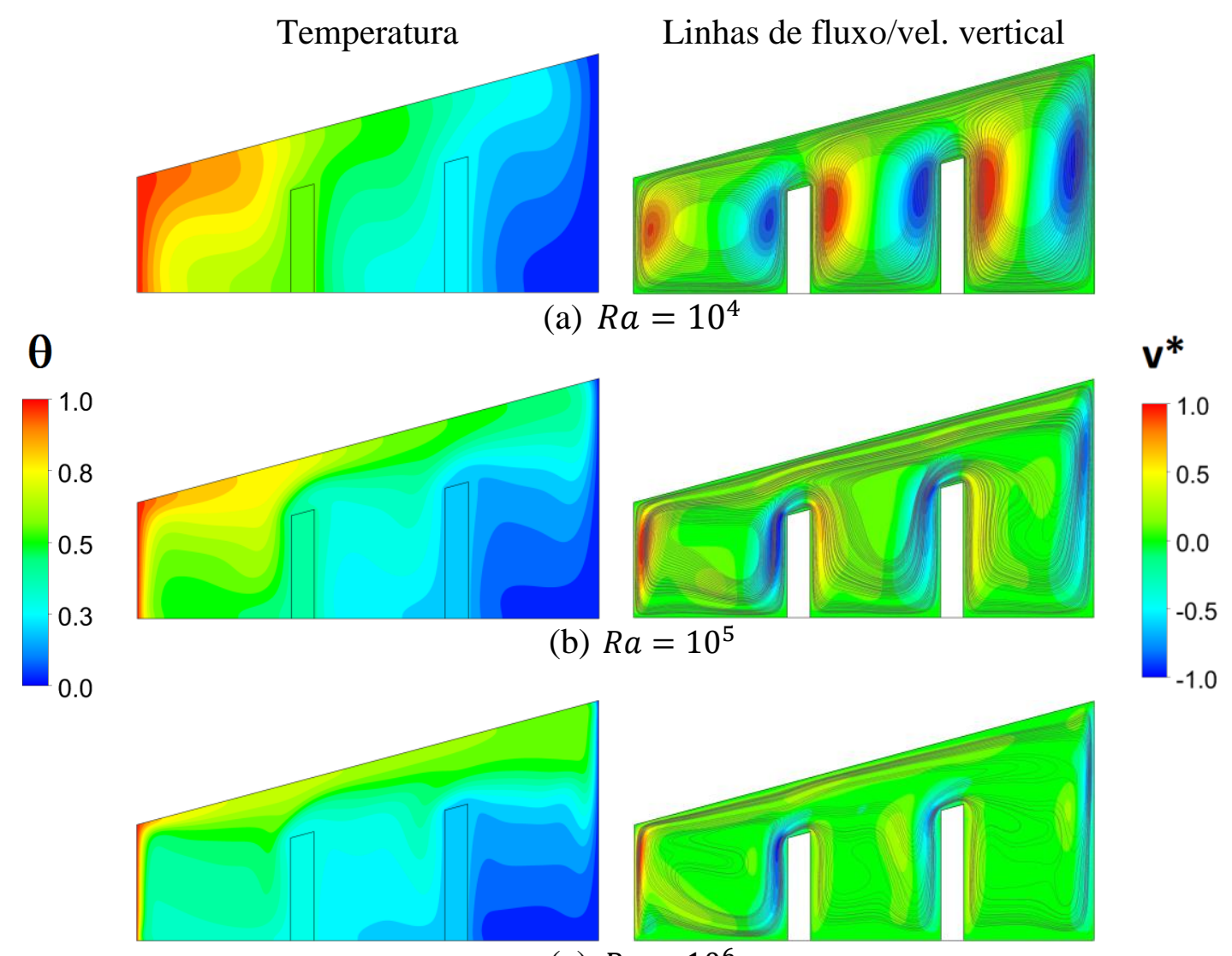

(c) $R a=10^{6}$

Figura 2 - Perfis de temperatura e linhas de fluxo para diferentes valores de Ra, sem considerar troca térmica por radiação.

Para o Caso 2, observado na Fig. 3, em que é considerada a presença da radiação somente na fase gasosa, pode-se comparar os resultados encontrados com a Fig. 2-b, é possível observar uma alteração significativa nos perfis de velocidade e temperatura, em particular na Fig. 3-a, caso em que a radiação é da mesma ordem que a convecção, pois $B o=1$. Além disso, ao aumentar os valores de $B o$, que simboliza uma relação entre convecção e radiação, ou seja, quanto menor seu valor, maior a influência da radiação no sistema, os resultados tendem a se aproximar dos encontrados quando a radiação era desconsiderada (Caso1). Quanto aos perfis de temperatura encontrados, constata-se que a presença da radiação diminui a estratificação destes, gerando um padrão em que as regiões próximas às paredes esquerdas sejam, em média, mais quentes que as 
regiões adjacentes às paredes direitas, uma demonstração mais concreta disso é vista na Fig. 3-a, na qual é possível contemplar um gradiente de temperatura maior ao longo do obstáculo devido à absorção e emissão de radiação pela superfície desse. Ademais, como consequência da alteração nos perfis de temperatura, os perfis de velocidade também são alterados. Para $B o=1$ (Fig. 3-a), ocorre o surgimento de uma região estagnada entre os dois defletores, à medida que para valores superiores do número de Boltzmann (Fig. 3-b e Fig. 3-c), os perfis em cada uma das três regiões separadas pelos defletores dispõe de uma maior simetria.

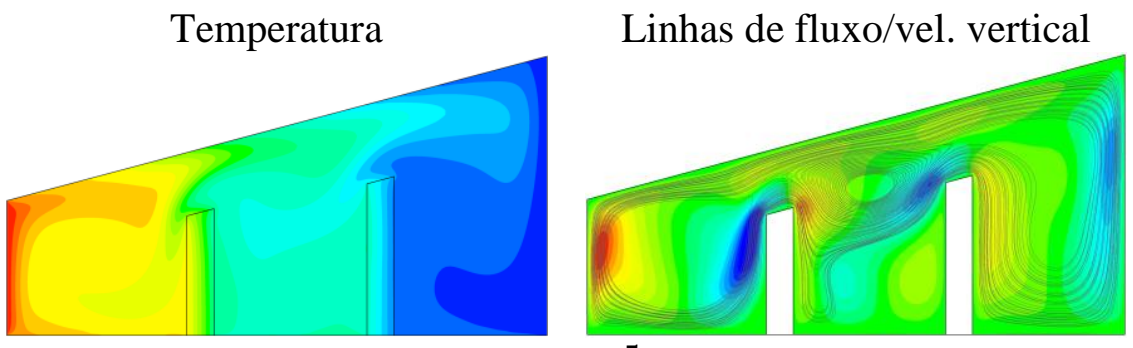

(a) $R a=10^{5}, B o=1$
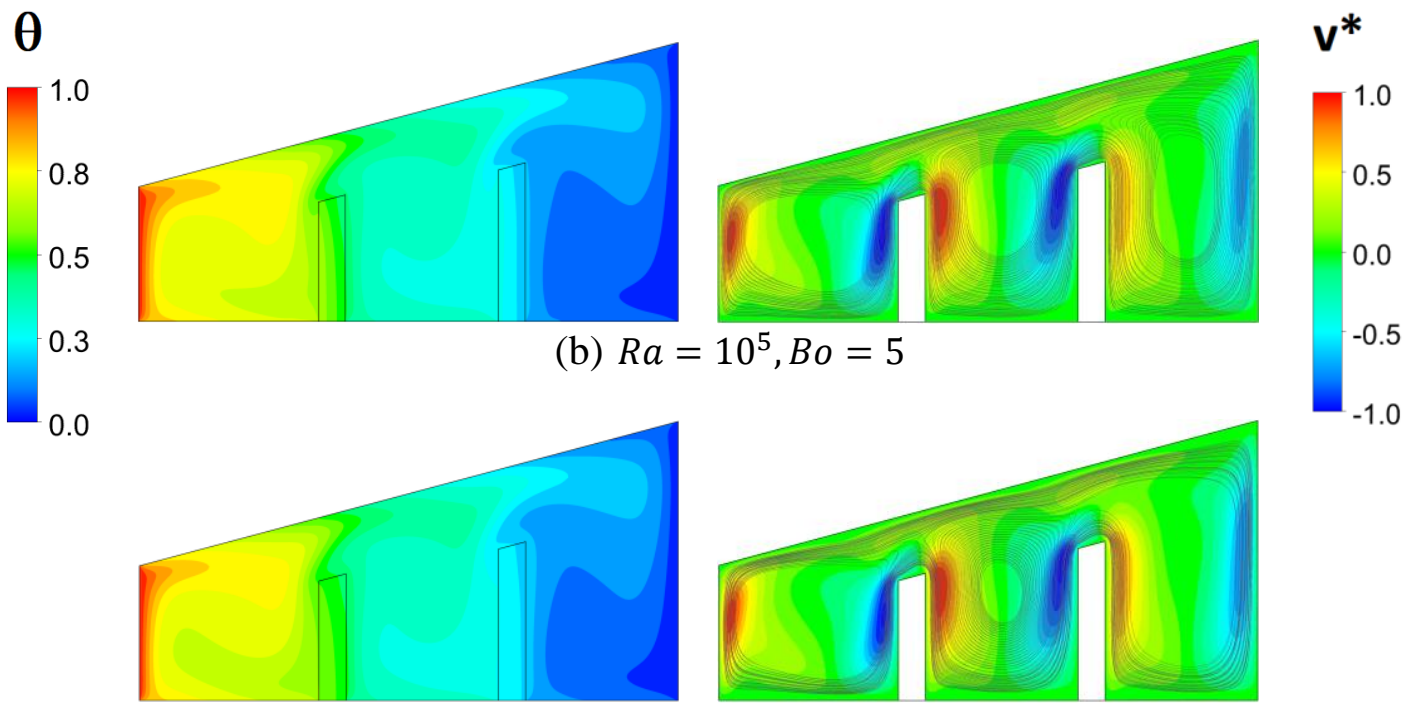

(c) $R a=10^{5}, B o=10$

Figura 3 - Perfis de temperatura e linhas de fluxo para $R a=10^{5}$ e diferentes valores de $B o$, considerando radiação na fase gasosa e obstáculos opacos.

Ao estudar-se os perfis encontrados no Caso 3, em que é avaliada a convecção, condução e radiação tanto para a fase gasosa, quanto para os obstáculos, utilizando o modelo de Monte Carlo para a fase sólida, encontrou-se os resultados dispostos na Fig. 4. Devido à passagem de radiação na fase sólida, são observados perfis similares para os diferentes valores de $B o$, atentando-se para os perfis de velocidade que se apresentam praticamente idênticos entre si e se assemelhando-se ao verificado no Caso 2, Fig. 2-b, para um alto valor de $B o$.

No caso discutido anteriormente (Fig. 3), a superfície do sólido podia absorver e emitir calor por radiação, contudo a transferência no obstáculo ocorria somente por condução. Ao considerar-se um material semitransparente, como por exemplo um vidro, verifica-se que em decorrência do aumento da taxa de transferência, o gradiente de temperatura no sólido diminui, diferentemente da estratificação discutida para a Fig. 2-a. Além disso é notada uma maior 
irregularidade na distribuição da temperatura no sólido em razão ao aumento da relevância das variações locais no coeficiente convectivo e na radiação emitida ou absorvida.

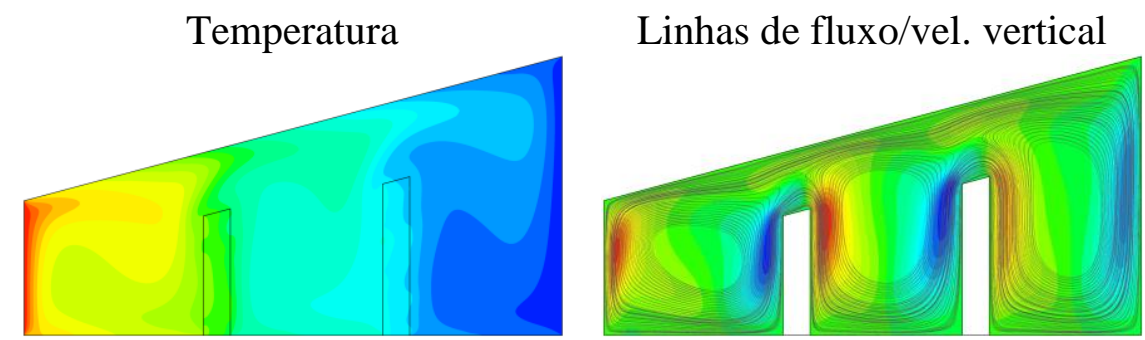

(a) $R a=10^{5}, B o=1$
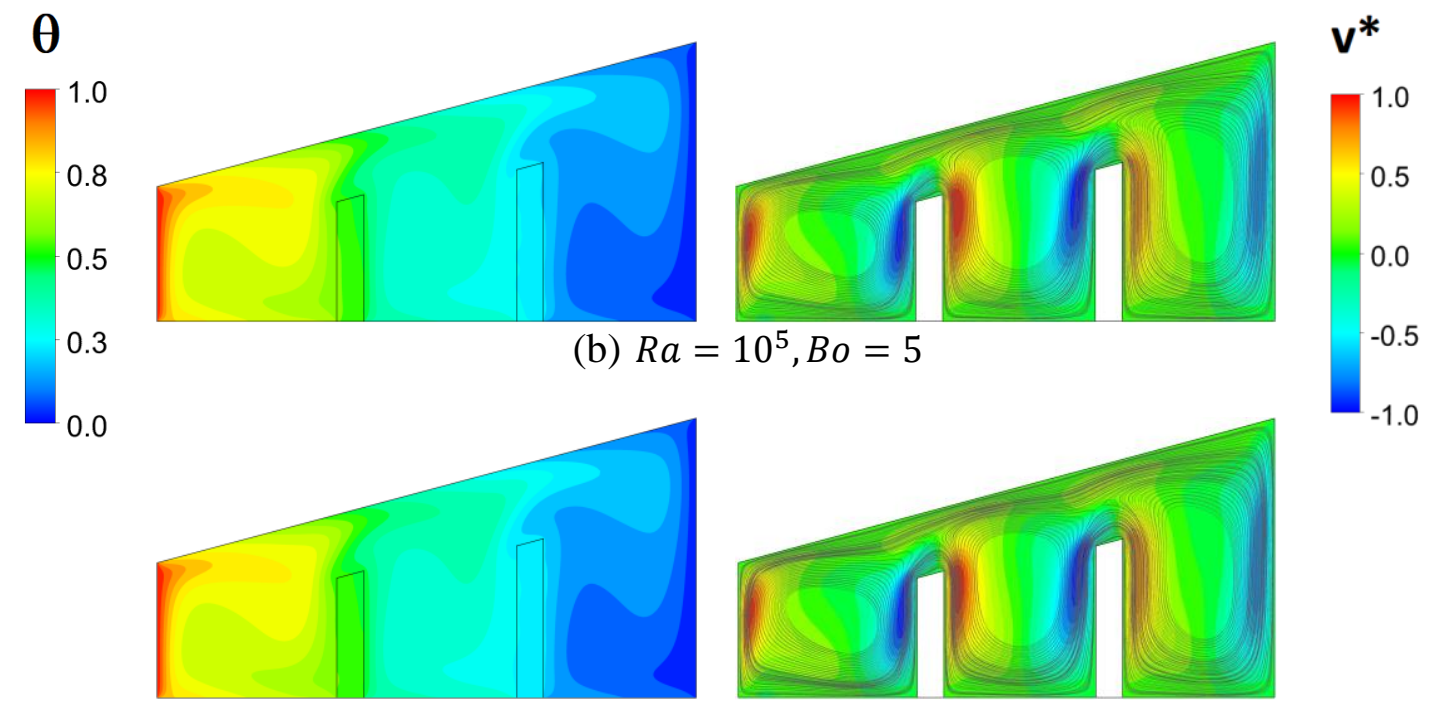

(c) $R a=10^{5}, B o=10$

Figura 4 - Perfis de temperatura e linhas de fluxo para $R a=10^{5}$ e diferentes valores de $B o$, considerando radiação nas fases sólida e gasosa e obstáculos semitransparentes.

\section{Conclusão}

O presente artigo investigou o efeito da radiação na convecção natural de cavidades trapezoidais equipadas com dois defletores. A cavidade modelada possui paredes laterais isotérmicas, sendo a esquerda mantida aquecida, enquanto a direita manteve-se em uma temperatura inferior, à medida que as superfícies de topo e fundo foram mantidas isoladas. As equações foram resolvidas utilizando o método de volumes finitos com o uso do pacote ANSYS CFX versão 16.0. Foram investigados três diferentes casos; primeiramente a troca térmica sem radiação; logo após supondo a convecção e radiação somente para a fase gasosa e, por último avaliando a convecção, condução e radiação tanto para a fase gasosa, quanto para a fase sólida.

Averiguou-se que para elevados valores de Rayleigh, a troca térmica por meio da condução perde espaço para a convecção. Também é possível concluir que a radiação faz com que os perfis de temperatura sejam menos estratificados, além disso constatou-se que para valores elevados do número de Boltzmann os perfis em cada uma das três regiões separadas pelos defletores são mais simétricos.

No caso em que a radiação é analisada em ambas as fases, demandou-se um gasto computacional muito maior do que os anteriores, devido a grande complexidade de seu 
modelamento. Neste conjunto também foi observado uma redução no gradiente de temperatura no sólido, sendo também a distribuição de temperatura mais irregular. Ademais detectou-se que mesmo para diferentes números de Boltzmann os perfis encontrados são semelhantes, diferentemente de quando a radiação era somente avaliada no fluido, neste contexto, a influência do número de Boltzmann era perceptível, sendo detectado até a presença de zonas estagnas para seus valores mais baixos.

\section{Agradecimentos}

Os autores prestam agradecimentos ao CNPq (Conselho Nacional de Desenvolvimento Científico e Tecnológico do Brasil) pelo apoio financeiro através do processo 407625/2016-0.

\section{Referências}

1. Incropera, F. P.; DeWitt D. P.; Bergman, T. L.; Lavine, A. S. Fundamentals of heat and mass transfer, LTC, Rio de Janeiro, 2008.

2. Fontana, É., Ph.D. Tese, Universidade Federal de Santa Catarina, 2014.

3. Silva, A.; Fontana, É.; Mariani V. C.; Marcondes, F. Numerical investigation of several physical and geometric parameters in the natural convection into trapezoidal cavities. Internation Journal of Heat and Mass Transfer. 2012, 55, 6808-6818.

4. Moukalled, F. ; Acharya, S. Natural Convection In Trapezoidal Cavities With Baffles Mounted On The Upper Inclined Surfaces. Numerical Heat Transfer, Part A: Applications, 2010, 545-565.

5. Fontana, É.; Silva, A.; Marcondes, F.; Mariani, V. C. Natural Convection within Trapezoidal Enclosure with Two Baffles: Effect of Various Angles of Inclination. 21st Brazilian Congress of Mechanical Engineering, 2011.

6. Reynolds, D. J.; Morrison, G. L.; Behnia, M. Combined Radiation and

Natural Convection in a Trapezoidal Cavity Absorber. Solar Energy 76, 2004, 229-234.

7. User Guide for ANSYS CFX Release 16.0, ANSYS Inc Ltd 